\title{
Remarks on Some Inequalities for Analogues of the Polygamma Function
}

\author{
Kwara Nantomah
}

(Communicated by Mohammad MURSALEEN)

\begin{abstract}
The purpose of this study is twofold. The first is, to point out drawbacks of some recent results concerning analogues the Polygamma function. The second is to resolve the drawbacks by providing improvements of the previous results.
\end{abstract}

Keywords: Polygamma function, analogues, inequality.

AMS Subject Classification (2010): Primary: 33B15 ; Secondary: $26 D 15$.

\section{Introduction}

We begin by recalling the following definitions which will be used in the sequel.

The Polygamma function, $\psi^{(m)}(x)$ is defined for $m \in \mathbb{N}$ and $x>0$ by

$$
\begin{aligned}
\psi^{(m)}(x)=\frac{d^{m}}{d x^{m}} \psi(x) & =(-1)^{m+1} \sum_{n=0}^{\infty} \frac{m !}{(n+x)^{m+1}} \\
& =(-1)^{m+1} \int_{0}^{\infty} \frac{t^{m} e^{-x t}}{1-e^{-t}} d t
\end{aligned}
$$

where $\psi^{(0)}(x) \equiv \psi(x)$, and $\psi(x)$ is the Digamma function.

The $p$-analogue of the Polygamma function, $\psi_{p}^{(m)}(x)$ or in short, the $p$-Polygamma function is defined for $p \in \mathbb{N}$ and $x>0$ by (See [2], [4])

$$
\begin{aligned}
\psi_{p}^{(m)}(x) & =(-1)^{m-1} \sum_{n=0}^{p} \frac{m !}{(n+x)^{m+1}} \\
& =(-1)^{m+1} \int_{0}^{\infty} \frac{t^{m} e^{-x t}}{1-e^{-t}}\left(1-e^{-(p+1) t}\right) d t
\end{aligned}
$$

where $\psi_{p}^{(m)}(x) \rightarrow \psi^{(m)}(x)$ as $p \rightarrow \infty$.

The $q$-Polygamma function, $\psi_{q}^{(m)}(x)$ is also defined for $q \in(0,1)$ and $x>0$ by (See [1], [8] and the references therein)

$$
\begin{aligned}
\psi_{q}^{(m)}(x) & =(\ln q)^{m+1} \sum_{n=1}^{\infty} \frac{n^{m} q^{n x}}{1-q^{n}} \\
& =(-1)^{m+1} \int_{0}^{\infty} \frac{t^{m} e^{-x t}}{1-e^{-t}} d \mu_{q}(t)
\end{aligned}
$$

Received : 27-08-2016, Accepted : 05-01-2018 
where $\psi_{q}^{(m)}(x) \rightarrow \psi^{(m)}(x)$ as $q \rightarrow 1, \mu_{q}(t)=-\ln q \sum_{k=1}^{\infty} \delta(t+k \ln q)$ is a discrete measure and $\delta(t)$ is the Dirac delta function.

Then the ( $p, q)$-Polygamma function, $\psi_{p, q}^{(m)}(x)$ is also defined for $q \in(0,1), k>0$ and $x>0$ by (See [5], [7])

$$
\begin{aligned}
\psi_{p, q}^{(m)}(x) & =(\ln q)^{m+1} \sum_{n=1}^{p} \frac{n^{m} q^{n x}}{1-q^{n}} \\
& =(-1)^{m+1} \int_{0}^{\infty} \frac{t^{m} e^{-x t}}{1-e^{-t}}\left(1-e^{-(p+1) t}\right) d \mu_{q}(t)
\end{aligned}
$$

where $\psi_{p, q}^{(m)}(x) \rightarrow \psi_{q}^{(m)}(x)$ as $p \rightarrow \infty$, and $\psi_{p, q}^{(m)}(x) \rightarrow \psi_{p}^{(m)}(x)$ as $q \rightarrow 1$.

Also, the $(q, k)$-Polygamma function, $\psi_{q, k}^{(m)}(x)$ is defined for $q \in(0,1), k>0$ and $x>0$ by (See [6])

$$
\psi_{q, k}^{(m)}(x)=(\ln q)^{m+1} \sum_{n=1}^{\infty} \frac{(n k)^{m} q^{n k x}}{1-q^{n k}}
$$

where $\psi_{q, k}^{(m)}(x) \rightarrow \psi_{k}^{(m)}(x)$ as $q \rightarrow 1$, and $\psi_{q, k}^{(m)}(x) \rightarrow \psi_{q}^{(m)}(x)$ as $k \rightarrow 1$.

Recently, the following results were established among others for the various analogues of the Polygamma function.

Theorem 1.1 ([9], Theorem 2.2). For $n=1,2,3, \ldots$,

$$
\psi_{q}^{\left(\frac{m}{a}+\frac{n}{b}\right)}\left(\frac{x}{a}+\frac{y}{b}\right) \leq\left(\psi_{q}^{(m)}(x)\right)^{\frac{1}{a}}\left(\psi_{q}^{(n)}(y)\right)^{\frac{1}{b}}
$$

where $\frac{m+n}{2}$ is an integer, $a>1, \frac{1}{a}+\frac{1}{b}=1$.

Theorem 1.2 ([9], Theorem 2.3). For $n=1,2,3, \ldots$,

$$
\left(\psi_{q}^{(m)}(x)+\psi_{q}^{(n)}(y)\right)^{\frac{1}{u}} \leq\left(\psi_{q}^{(m)}(x)\right)^{\frac{1}{u}}+\left(\psi_{q}^{(n)}(y)\right)^{\frac{1}{u}}
$$

where $\frac{m+n}{2}$ is an integer, $u \geq 1$.

Theorem 1.3 ([4], Theorem 2.1). For $n=1,2,3, \ldots$,

$$
\psi_{p}^{\left(\frac{m}{a}+\frac{n}{b}\right)}\left(\frac{x}{a}+\frac{y}{b}\right) \leq\left(\psi_{p}^{(m)}(x)\right)^{\frac{1}{a}}\left(\psi_{p}^{(n)}(y)\right)^{\frac{1}{b}}
$$

where $\frac{m+n}{2}$ is an integer, $a>1, \frac{1}{a}+\frac{1}{b}=1$.

Theorem 1.4 ([4], Theorem 2.3). For $n=1,2,3, \ldots$,

$$
\left(\psi_{p}^{(m)}(x)+\psi_{p}^{(n)}(y)\right)^{\frac{1}{u}} \leq\left(\psi_{p}^{(m)}(x)\right)^{\frac{1}{u}}+\left(\psi_{p}^{(n)}(y)\right)^{\frac{1}{u}}
$$

where $\frac{m+n}{2}$ is an integer, $u \geq 1$.

Theorem 1.5 ([5], Theorem 2.1). For $n=1,2,3, \ldots$,

$$
\psi_{p, q}^{\left(\frac{m}{a}+\frac{n}{b}\right)}\left(\frac{x}{a}+\frac{y}{b}\right) \leq\left(\psi_{p, q}^{(m)}(x)\right)^{\frac{1}{a}}\left(\psi_{p, q}^{(n)}(y)\right)^{\frac{1}{b}}
$$

where $\frac{m+n}{2}$ is an integer, $a>1, \frac{1}{a}+\frac{1}{b}=1$.

Theorem 1.6 ([5], Theorem 2.2). For $n=1,2,3, \ldots$,

$$
\left(\psi_{p, q}^{(m)}(x)+\psi_{p, q}^{(n)}(y)\right)^{\frac{1}{u}} \leq\left(\psi_{p, q}^{(m)}(x)\right)^{\frac{1}{u}}+\left(\psi_{p, q}^{(n)}(y)\right)^{\frac{1}{u}}
$$

where $\frac{m+n}{2}$ is an integer, $u \geq 1$. 
Theorem 1.7 ([6], Theorem 2.1). For $n=1,2,3, \ldots$,

$$
\psi_{q, k}^{\left(\frac{m}{a}+\frac{n}{b}\right)}\left(\frac{x}{a}+\frac{y}{b}\right) \leq\left(\psi_{q, k}^{(m)}(x)\right)^{\frac{1}{a}}\left(\psi_{q, k}^{(n)}(y)\right)^{\frac{1}{b}}
$$

where $\frac{m+n}{2}$ is an integer, $a>1, \frac{1}{a}+\frac{1}{b}=1$.

Theorem 1.8 ([6], Theorem 2.3). For $n=1,2,3, \ldots$,

$$
\left(\psi_{q, k}^{(m)}(x)+\psi_{q, k}^{(n)}(y)\right)^{\frac{1}{u}} \leq\left(\psi_{q, k}^{(m)}(x)\right)^{\frac{1}{u}}+\left(\psi_{q, k}^{(n)}(y)\right)^{\frac{1}{u}}
$$

where $\frac{m+n}{2}$ is an integer, $u \geq 1$.

Nonetheless, these elegant inequalities do have some drawbacks. They do not hold for even $m$ and $n$. Notice from (1.1), (1.2), (1.3) and (1.4) that, for even $m$,

$$
\psi_{q}^{(m)}(x)<0, \quad \psi_{p}^{(m)}(x)<0, \quad \psi_{q, k}^{(m)}(x)<0 \quad \text { and } \quad \psi_{p, q}^{(m)}(x)<0 .
$$

Consequently, if $a=b=u=2$ in particular, the quantities

$$
\left(\psi_{q}^{(m)}(x)\right)^{\frac{1}{2}}, \quad\left(\psi_{p}^{(m)}(x)\right)^{\frac{1}{2}}, \quad\left(\psi_{q, k}^{(m)}(x)\right)^{\frac{1}{2}} \text { and }\left(\psi_{p, q}^{(m)}(x)\right)^{\frac{1}{2}}
$$

are not defined since they have to be real. Also, since $\frac{m}{a}+\frac{n}{b}$ is an order of a derivative, there is the need to add to the hypotheses of Theorems 1.1, 1.3, 1.5 and 1.7 that $\frac{m}{a}+\frac{n}{b} \in \mathbb{N}$. Furthermore, in the proofs of Theorems 1.2, 1.4, 1.6 and 1.8, the authors relied on the fact that $\alpha^{u}+\beta^{u} \leq(\alpha+\beta)^{u}$, for $\alpha, \beta \geq 0$ and $u \geq 1$. However, for even $m$ and $n$, the requirement that $\alpha, \beta \geq 0$ was overlooked in the proofs.

In the following section, we resolve these challenges by providing improvements of the above results. We utilize the techniques of the previous authors.

\section{Improvements}

Theorem 2.1. Let $m, n \in \mathbb{N}, a>1, \frac{1}{a}+\frac{1}{b}=1$ such that $\frac{m}{a}+\frac{n}{b} \in \mathbb{N}$. Then, the inequality

$$
\left|\psi_{p, q}^{\left(\frac{m}{a}+\frac{n}{b}\right)}\left(\frac{x}{a}+\frac{y}{b}\right)\right| \leq\left|\psi_{p, q}^{(m)}(x)\right|^{\frac{1}{a}}\left|\psi_{p, q}^{(n)}(y)\right|^{\frac{1}{b}}
$$

holds for $x, y>0$.

Proof. We use the Hölder's inequality for integrals. Then by (1.3) we obtain

$$
\begin{aligned}
\left|\psi_{p, q}^{\left(\frac{m}{a}+\frac{n}{b}\right)}\left(\frac{x}{a}+\frac{y}{b}\right)\right|= & \int_{0}^{\infty} \frac{1-e^{-(p+1) t}}{1-e^{-t}} t^{\left(\frac{m}{a}+\frac{n}{b}\right)} e^{-\left(\frac{x}{a}+\frac{y}{b}\right) t} d \mu_{q}(t) \\
& =\int_{0}^{\infty}\left(\frac{1-e^{-(p+1) t}}{1-e^{-t}}\right)^{\frac{1}{a}} t^{\frac{m}{a}} e^{-\frac{x t}{a}}\left(\frac{1-e^{-(p+1) t}}{1-e^{-t}}\right)^{\frac{1}{b}} t^{\frac{n}{b}} e^{-\frac{y t}{b}} d \mu_{q}(t) \\
& \leq\left(\int_{0}^{\infty} \frac{1-e^{-(p+1) t}}{1-e^{-t}} t^{m} e^{-x t} d \mu_{q}(t)\right)^{\frac{1}{a}} \\
& \times\left(\int_{0}^{\infty} \frac{1-e^{-(p+1) t}}{1-e^{-t}} t^{n} e^{-y t} d \mu_{q}(t)\right)^{\frac{1}{b}} \\
& =\left|\psi_{p, q}^{(m)}(x)\right|^{\frac{1}{a}}\left|\psi_{p, q}^{(n)}(y)\right|^{\frac{1}{b}} .
\end{aligned}
$$


Theorem 2.2. Let $m, n \in \mathbb{N}$ and $u \geq 1$. Then, the inequality

$$
\left(\left|\psi_{p, q}^{(m)}(x)\right|+\left|\psi_{p, q}^{(n)}(y)\right|\right)^{\frac{1}{u}} \leq\left|\psi_{p, q}^{(m)}(x)\right|^{\frac{1}{u}}+\left|\psi_{p, q}^{(n)}(y)\right|^{\frac{1}{u}}
$$

holds for $x, y>0$.

Proof. We used the fact that $\alpha^{u}+\beta^{u} \leq(\alpha+\beta)^{u}$, for $\alpha, \beta \geq 0, u \geq 1$, and the Minkowski's inequality for integral. Then by (1.3), we obtain

$$
\begin{aligned}
& \left(\left|\psi_{p, q}^{(m)}(x)\right|+\left|\psi_{p, q}^{(n)}(y)\right|\right)^{\frac{1}{u}} \\
& =\left(\int_{0}^{\infty} \frac{1-e^{-(p+1) t}}{1-e^{-t}} t^{m} e^{-x t} d \mu_{q}(t)+\int_{0}^{\infty} \frac{1-e^{-(p+1) t}}{1-e^{-t}} t^{n} e^{-y t} d \mu_{q}(t)\right)^{\frac{1}{u}} \\
& =\left(\int_{0}^{\infty}\left[\left(\left(\frac{1-e^{-(p+1) t}}{1-e^{-t}}\right)^{\frac{1}{u}} t^{\frac{m}{u}} e^{\frac{-x t}{u}}\right)^{u}+\left(\left(\frac{1-e^{-(p+1) t}}{1-e^{-t}}\right)^{\frac{1}{u}} t^{\frac{n}{u}} e^{\frac{-y t}{u}}\right)^{u}\right] d \mu_{q}(t)\right)^{\frac{1}{u}} \\
& \leq\left(\int_{0}^{\infty}\left[\left(\left(\frac{1-e^{-(p+1) t}}{1-e^{-t}}\right)^{\frac{1}{u}} t^{\frac{m}{u}} e^{\frac{-x t}{u}}\right)+\left(\left(\frac{1-e^{-(p+1) t}}{1-e^{-t}}\right)^{\frac{1}{u}} t^{\frac{n}{u}} e^{\frac{-y t}{u}}\right)\right]^{u} d \mu_{q}(t)\right)^{\frac{1}{u}} \\
& \leq\left(\int_{0}^{\infty} \frac{1-e^{-(p+1) t}}{1-e^{-t}} t^{m} e^{-x t} d \mu_{q}(t)\right)^{\frac{1}{u}}+\left(\int_{0}^{\infty} \frac{1-e^{-(p+1) t}}{1-e^{-t}} t^{n} e^{-y t} d \mu_{q}(t)\right)^{\frac{1}{u}} \\
& =\left|\psi_{p, q}^{(m)}(x)\right|^{\frac{1}{u}}+\left|\psi_{p, q}^{(n)}(y)\right|^{\frac{1}{u}} \text {. }
\end{aligned}
$$

Theorem 2.3. Let $m, n \in \mathbb{N}, a>1, \frac{1}{a}+\frac{1}{b}=1$ such that $\frac{m}{a}+\frac{n}{b} \in \mathbb{N}$. Then, the inequality

$$
\left|\psi_{q, k}^{\left(\frac{m}{a}+\frac{n}{b}\right)}\left(\frac{x}{a}+\frac{y}{b}\right)\right| \leq\left|\psi_{q, k}^{(m)}(x)\right|^{\frac{1}{a}}\left|\psi_{q, k}^{(n)}(y)\right|^{\frac{1}{b}}
$$

holds for $x, y>0$.

Proof. By (1.4) and the Hölder's inequality for sums, we obtain

$$
\begin{aligned}
\left|\psi_{q, k}^{\left(\frac{m}{a}+\frac{n}{b}\right)}\left(\frac{x}{a}+\frac{y}{b}\right)\right| & =|\ln q|^{\left(\frac{m}{a}+\frac{n}{b}\right)+1} \sum_{s=1}^{\infty} \frac{(s k)^{\left(\frac{m}{a}+\frac{n}{b}\right)} q^{s k\left(\frac{x}{a}+\frac{y}{b}\right)}}{1-q^{s k}} \\
& =|\ln q|^{\frac{m+1}{a}}|\ln q|^{\frac{n+1}{b}} \sum_{s=1}^{\infty} \frac{(s k)^{\frac{m}{a}} q^{\frac{s k x}{a}}}{\left(1-q^{s k}\right)^{\frac{1}{a}}} \cdot \frac{(s k)^{\frac{n}{b}} q^{\frac{s k y}{b}}}{\left(1-q^{s k}\right)^{\frac{1}{b}}} \\
& \leq\left(|\ln q|^{m+1} \sum_{s=1}^{\infty} \frac{(s k)^{m} q^{s k x}}{1-q^{s k}}\right)^{\frac{1}{a}}\left(|\ln q|^{n+1} \sum_{s=1}^{\infty} \frac{(s k)^{n} q^{s k y}}{1-q^{s k}}\right)^{\frac{1}{b}} \\
& =\left|\psi_{q, k}^{(m)}(x)\right|^{\frac{1}{a}}\left|\psi_{q, k}^{(n)}(y)\right|^{\frac{1}{b}} .
\end{aligned}
$$

Theorem 2.4. Let $m, n \in \mathbb{N}$ and $u \geq 1$. Then, the inequality

$$
\left(\left|\psi_{q, k}^{(m)}(x)\right|+\left|\psi_{q, k}^{(n)}(y)\right|\right)^{\frac{1}{u}} \leq\left|\psi_{q, k}^{(m)}(x)\right|^{\frac{1}{u}}+\left|\psi_{q, k}^{(n)}(y)\right|^{\frac{1}{u}}
$$

holds for $x, y>0$. 
Proof. Here too, we use the Minkowski's inequality for sums and the fact that $\alpha^{u}+\beta^{u} \leq(\alpha+\beta)^{u}$, for $\alpha, \beta \geq 0$, $u \geq 1$. Then from (1.4) we obtain

$$
\begin{aligned}
& \left(\left|\psi_{q, k}^{(m)}(x)\right|+\left|\psi_{q, k}^{(n)}(y)\right|\right)^{\frac{1}{u}} \\
& =\left(|\ln q|^{m+1} \sum_{s=1}^{\infty} \frac{(s k)^{m} q^{s k x}}{1-q^{s k}}+|\ln q|^{n+1} \sum_{s=1}^{\infty} \frac{(s k)^{n} q^{s k y}}{1-q^{s k}}\right)^{\frac{1}{u}} \\
& =\left(\sum_{s=1}^{\infty}\left[\left(|\ln q|^{\frac{m+1}{u}} \frac{(s k)^{\frac{m}{u}} q^{\frac{s k x}{u}}}{\left(1-q^{s k}\right)^{\frac{1}{u}}}\right)^{u}+\left(|\ln q|^{\frac{n+1}{u}} \frac{(s k)^{\frac{n}{u}} q^{\frac{s k y}{u}}}{\left(1-q^{s k}\right)^{\frac{1}{u}}}\right)^{u}\right]\right)^{\frac{1}{u}} \\
& \leq\left(\sum_{s=1}^{\infty}\left[\left(|\ln q|^{\frac{m+1}{u}} \frac{(s k)^{\frac{m}{u}} q^{\frac{s k x}{u}}}{\left(1-q^{s k}\right)^{\frac{1}{u}}}\right)+\left(|\ln q|^{\frac{n+1}{u}} \frac{(s k)^{\frac{n}{u}} q^{\frac{s k y}{u}}}{\left(1-q^{s k}\right)^{\frac{1}{u}}}\right)\right]^{u}\right)^{\frac{1}{u}} \\
& \leq\left(|\ln q|^{m+1} \sum_{s=1}^{\infty} \frac{(s k)^{m} q^{s k x}}{1-q^{s k}}\right)^{\frac{1}{u}}+\left(|\ln q|^{n+1} \sum_{s=1}^{\infty} \frac{(s k)^{n} q^{s k y}}{1-q^{s k}}\right)^{\frac{1}{u}} \\
& =\left|\psi_{q, k}^{(m)}(x)\right|^{\frac{1}{u}}+\left|\psi_{q, k}^{(n)}(y)\right|^{\frac{1}{u}} .
\end{aligned}
$$

Theorem 2.5. Let $m, n \in \mathbb{N}, a>1, \frac{1}{a}+\frac{1}{b}=1$ such that $\frac{m}{a}+\frac{n}{b} \in \mathbb{N}$. Then, the inequality

$$
\left|\psi_{q}^{\left(\frac{m}{a}+\frac{n}{b}\right)}\left(\frac{x}{a}+\frac{y}{b}\right)\right| \leq\left|\psi_{q}^{(m)}(x)\right|^{\frac{1}{a}}\left|\psi_{q}^{(n)}(y)\right|^{\frac{1}{b}}
$$

holds for $x, y>0$.

Proof. Let $p \rightarrow \infty$ in Theorem 2.1 or let $k \rightarrow 1$ in Theorem 2.3.

Theorem 2.6. Let $m, n \in \mathbb{N}$ and $u \geq 1$. Then, the inequality

$$
\left(\left|\psi_{q}^{(m)}(x)\right|+\left|\psi_{q}^{(n)}(y)\right|\right)^{\frac{1}{u}} \leq\left|\psi_{q}^{(m)}(x)\right|^{\frac{1}{u}}+\left|\psi_{q}^{(n)}(y)\right|^{\frac{1}{u}}
$$

holds for $x, y>0$.

Proof. Let $p \rightarrow \infty$ in Theorem 2.2 or let $k \rightarrow 1$ in Theorem 2.4.

Theorem 2.7. Let $m, n \in \mathbb{N}, a>1, \frac{1}{a}+\frac{1}{b}=1$ such that $\frac{m}{a}+\frac{n}{b} \in \mathbb{N}$. Then, the inequality

$$
\left|\psi_{p}^{\left(\frac{m}{a}+\frac{n}{b}\right)}\left(\frac{x}{a}+\frac{y}{b}\right)\right| \leq\left|\psi_{p}^{(m)}(x)\right|^{\frac{1}{a}}\left|\psi_{p}^{(n)}(y)\right|^{\frac{1}{b}}
$$

holds for $x, y>0$.

Proof. Let $q \rightarrow 1$ in Theorem 2.1.

Theorem 2.8. Let $m, n \in \mathbb{N}$ and $u \geq 1$. Then, the inequality

$$
\left(\left|\psi_{p}^{(m)}(x)\right|+\left|\psi_{p}^{(n)}(y)\right|\right)^{\frac{1}{u}} \leq\left|\psi_{p}^{(m)}(x)\right|^{\frac{1}{u}}+\left|\psi_{p}^{(n)}(y)\right|^{\frac{1}{u}}
$$

holds for $x, y>0$.

Proof. Let $q \rightarrow 1$ in Theorem 2.2.

\section{Conclusion}

In this paper, drawbacks of some recent results concerning analogues of the Polygamma function are pointed out and some improved results provided to resolve the drawbacks. 


\section{References}

[1] Ismail M. E. H. and Muldoon M. E., Higher Monotonicity Properties of $q$-gamma and $q$-psi Functions, $A d v$. Dyn. Syst. Appl., 8(2)(2013), 247-259.

[2] Krasniqi V. and Shabani A. S., Convexity Properties and Inequalities for a Generalized Gamma Function, Appl. Math. E-Notes, 10(2010), 27-35.

[3] Laforgia A. and Natalini P., Turan type inequalities for some special functions, J. Ineq. Pure Appl. Math., 7(1)(2006), Art. 22, 1-5.

[4] Merovci F., Turan type inequalities for p-Polygamma functions, Matematiche (Catania), LXVIII(II)(2013), 99-106.

[5] Merovci F., Turan type inequalities for $(p, q)$-Gamma function, Scientia Magna, 9(1)(2013), 25-30.

[6] Merovci F., Turan type inequalities for some ( $q, k)$-Special functions, Acta Univ. Apulensis Math. Inform., 34(2013), 69-76.

[7] Nantomah K., The $(p, q)$-Analogues of Some Inequalities for the Digamma Function, J. Inequal. Spec. Funct., $5(2)(2014), 10-14$.

[8] Qi F., A Completely Monotonic Function Related to the $q$-Trigamma Function, U.P.B. Sci. Bull., Series A, 76(1)(2014), 107-114.

[9] Sulaiman W. T., Turan type Inequalities for Some Special Functions, Aust. J. Math. Anal. Appl., 9(1)(2012), 1-7.

\section{Affiliations}

\section{KWARA NANTOMAH}

AdDress: Department of Mathematics, Faculty of Mathematical Sciences, University for Development Studies, Navrongo Campus, P. O. Box 24, Navrongo, UE/R, Ghana.

E-MAIL: mykwarasoft@yahoo.com, knantomah@uds.edu.gh

ORCID ID: $0000-0003-0911-9537$ 